\title{
The Relative Weight of the Organs of Dogs.
}

\author{
By \\ HIROSHI SATO. \\ (佐 藤 熙) \\ (From the Physiological Laboratory of Prof. Y. Sataké, \\ Tohohu Imperial University, Sendai.)
}

Accurate knowledge of the relative weight (compared to the body weight) of the organs in normal animals is not infrequently required as the control of that of animals dying under pathological conditions, natural as well as experimental. Of some organs we have some reliable information ; the measurement has usually been made of the normal animal for obtaining the standard on the occasion of studying some alteration in that organ. We have felt that the extensive work of $\mathrm{Brown}$ and his co-workers ${ }^{1)}$ on the weight of the organs in normal male rabbits, amounting to 644 , is to be highly appreciated. It not only affords to some extent reliable standard figures, but also the mutual relations between the weights of various organs. Sometimes it is advisable or rather indispensable to measure the normal standard value by one and the same hand as the experiment under question, as the relative weight (the organ weight in relation to the body weight) may vary according to the feeding, climate, etc., etc. However the values reached by other experimentalists, when they are based on a vast number of estimations, are certainly sufficient to use as the standard, or at least they may serve as the control for the control experiments made by one and the same hand but on a small number of animals, as not seldom happens.

The present writer has had opportunities to weigh the organs of a great number of dogs (in toto 252), which were well nourished and lilled by bleeding after conducting one simple experiment, which seemingly did not so seriously affect the organism.

1) Brown, Pearce and Van Allen, J. of Exp. Med., 1925, 42, 69; 1926, 43, 733. 
Some previous references may be cited here: In the book of Ellenberger and $\mathrm{Ba}$ a m on the anatomy of the dog, the weight of the liver, spleen, lungs and kidney of this species of animal is noted as $1 / 17-1 / 26,1 / 500-1 / 600,1 / 60-1 / 90$ and $1 / 140-1 / 185$ of the body weight respectively (viz. 58.8-38.5 grms., 2-1.7 grms., 16.6-11.1 grms. and 7.1-5.4 grms. per kilo of body weight). ${ }^{2}$ The number of cases examined is not related. The following data are quoted here from the results obtained by $\mathrm{J}$ un kersd o $\mathrm{f}^{3)}$ himself: Heart $0.79 \%$ of the body weight $(0.64-0.90 \% ; 6$ individuals), liver $2.66 \%(2.50-2.86 \%$; 5 individuals $)$, kidneys $0.47 \%(0.39-0.57 \% ; 7)$, pancreas $0.234(0.14-0.31 \% ; 7)$, spleen $0.197 \%(0.150-0.260 \% ; 4)$, thyroid gland $0.0235 \%(0.024 \& 0.023 \%)$ and suprarenal glands $0.0231 \%(0.017,0.017 \&$ $0.030 \%$ ). In 63 dogs of $\mathrm{K}$ u rak a m i, the mean, maximum and minimum weight of the pancreas of dogs, which was removed operatively, were $3.17 \mathrm{grms}$, $4.0 \mathrm{grms}$. and 2.8 grms. respectively. The most frequent mode $2.9-3.0-3.1 \mathrm{grms}$.

\section{Material and Method :}

252 dogs were examined between June 1925 and April 1929. They were all well nourished, and used in experiments on the sensory stimulation, adrenaline, B-tetra, guanidine, peptone, diphtheria toxine, coffeine, haemorrhage, cooling and heating, urethane, strychnine and the heart reflex (the blood pressure experiment), which have been previously reported in this Journal. The dogs employed by Suzuki in the study on the heart reflex make about one third of the animals utilized here. A few of the dogs, provided with the cava pocket, were also examined, but the kidneys were discarded on those occasions. Strictly speaking the animals included in this paper were by no means normal. Various breeds of dogs were included in the present series of experiments, and the age of the animals was estimated according to Boenish. In the majority of cases the animals were old, from six months to six years. In all the cases the dogs were killed finally by bleeding, and then immediately the organs removed. The organs looking apparently abnormal were discarded. Among the organs, the pancreas, the spleen, the liver, the lungs, the heart, the kidneys and the suprarenals were usually weighed. Rather recently the brain, the hypophysis and the thyroid were also weighed, but they are omitted from our report by reason of their small number.

The organs were prepared for weighing by trimming and removing the external tissucs, when existing. The vessels from and to the heart were

2) Ellenberger and B a u m, Anatomie des Hundes, Berlin 1891, 308, 311, 326, 335.

3) Junkersdorf, Pflüger's Arch., 1925, 210, 351.

4) Kurakami, Juralo Asoico med. Nagasaki (Jap.), 1925, 3, 177 (Abstract in German p. 16).

5) F. Boen isch, Arch. f. wiss. u. prakt. Tierheilk., 1913, 39, 289. 
cut off on the level of the auricles; the cardiac chambers were opened and freed from the blood coagula, etc. The trachea was cut on the level of the bifurcation and all the lung lobes were weighed together.

A balance of $1 \mathrm{grm}$. sensibility was employed for weighing the pancreas, spleen, liver, heart, lungs and kidneys, a chemical balance of 0.0005 grm. sensibility for the suprarenals and a balance of 0.1 kilo sensibility for the body weight.

The body weight varied from 3.5 kilos to 40.0 ; in the present paper not the absolute weight of the organs, but only the relative weight, that is the weight of the organs per unit (kilo) of body weight, will be dealt with.

The data yielded in the present investigations are epitomized in Table and Figs. 1-7.

The distribution curves given as the solid line in Figures are based on the use of a group interval of approximately one-tenth of the difference between the maximum and minimum weight of the organ. The curves obtained by plotting the results were smoothed by the formula $\frac{a+2 b+c}{4}$. The smoothed curve is graphed as the dotted line. The limits of the standard deviation and the probable error are marked at the bottom of the curve with the brackets, respectively solid and dotted.

\section{REsults :}

\section{Tabie I.}

Summary of numerical values obtained from determinations of organ weights made on 252 dogs of various ages and breeds between June, 1925 and April, 1929. The figures indicate the weight per kilo of body weight.

\begin{tabular}{|c|c|c|c|c|c|c|c|c|c|}
\hline Organ & 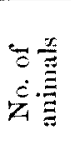 & 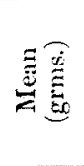 & 焉 & 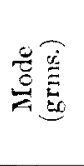 & 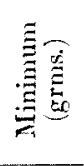 & 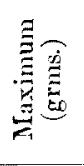 & 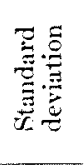 & $\begin{array}{l}\frac{5}{0} \\
\frac{1}{0} \\
\frac{0}{0} \\
0 \\
0\end{array}$ & 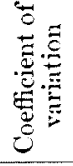 \\
\hline Heart & 225 & 8.0 & 7.9 & 7.5 & 4.0 & 11.2 & 1.204 & 0.812 & 15.05 \\
\hline Spleen & 248 & 3.8 & 3.5 & 2.8 & 1.0 & 8.6 & 1.476 & 0.995 & 38.8 \\
\hline Liver & 247 & 36 & 34 & 29 & 21 & 69 & 9.77 & 6.59 & 27.2 \\
\hline Pancreas & 243 & 2.7 & 2.6 & 2.8 & 1.1 & 5.7 & 0.831 & 0.560 & 30.8 \\
\hline Lungs & 226 & 10.1 & 9.3 & 8.3 & 5.0 & 22.8 & 3.09 & 2.08 & 30.6 \\
\hline Kidneys & 245 & 6.3 & 5.9 & 5.7 & 3.5 & 12.5 & 1.745 & 1.177 & 27.7 \\
\hline Suprarenals & 199 & 0.118 & 0.115 & 0.121 & 0.049 & 0.264 & 0.0375 & 0.0253 & 31.8 \\
\hline 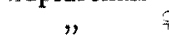 & 77 & 0.140 & 0.135 & & 0.057 & 0.264 & 0.0388 & 0.0262 & 27.7 \\
\hline$"$ & 122 & 0.104 & 0.100 & & 0.049 & 0.202 & 0.0810 & 0.0219 & 29.8 \\
\hline
\end{tabular}



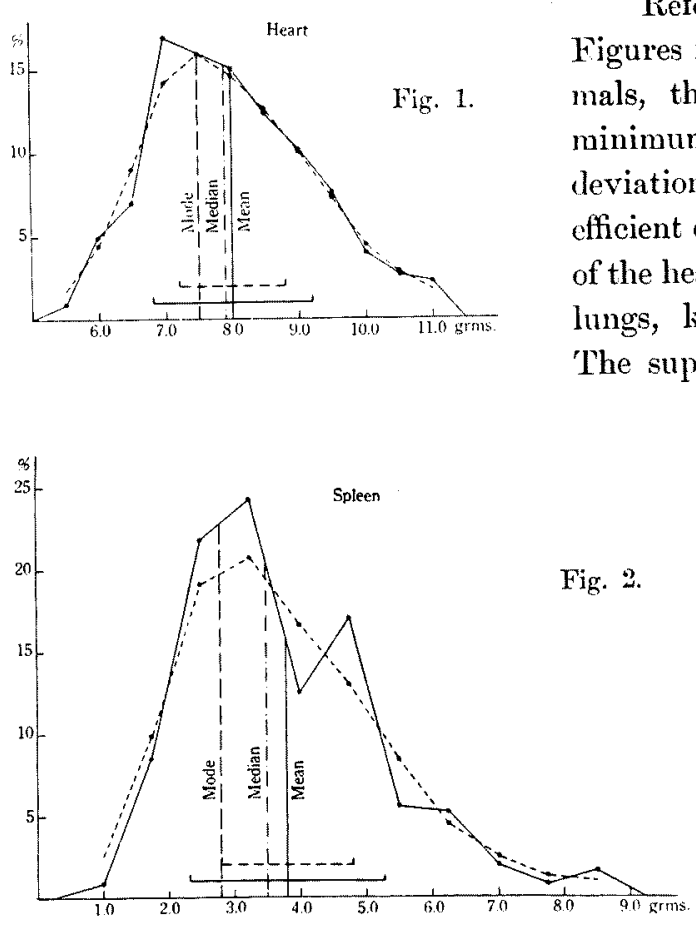

Reference to Table I and the Figures reveal the number of animals, the mean, median, mode, minimum, maximum, standard deviation, probable error and coefficient of variation of the weights of the heart, spleen, liver, pancreas, lungs, kidneys and suprarenals. The suprarenal glands were considered further according to the sex of the animals.

Each side of the kidneys and suprarenals were measured separately, but are given combined in the table and figures.

Of 243 dogs, both kidneys were similarly heavy in 47 cases, that is in $19 \%$, the left
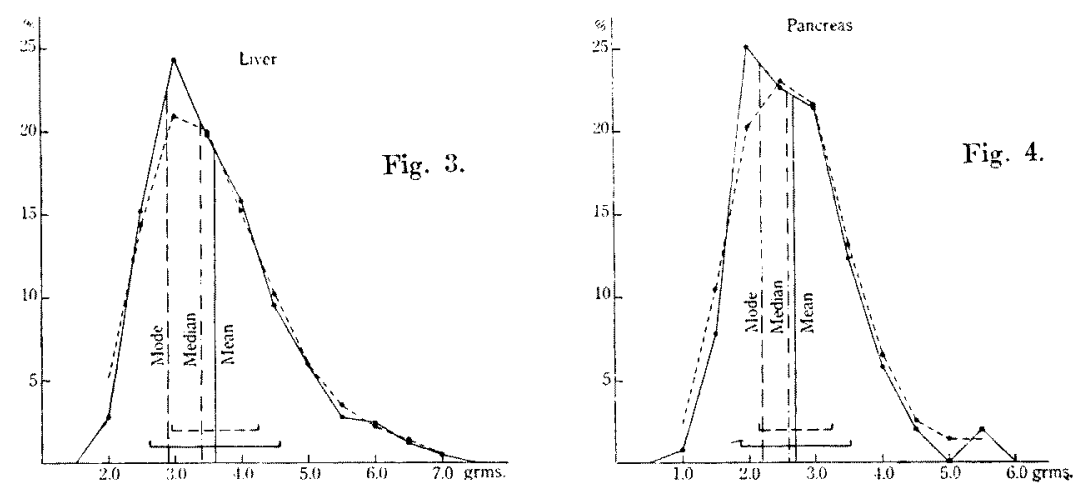

kidney heavier than the right in 150 cases, viz. $62 \%$, and the reverse held true in 46 cases viz. 19\%. In 150 cases, in which the left kidney was heavier than its fellow, the mean of the ratio (left : right) was 1.07:1 and 

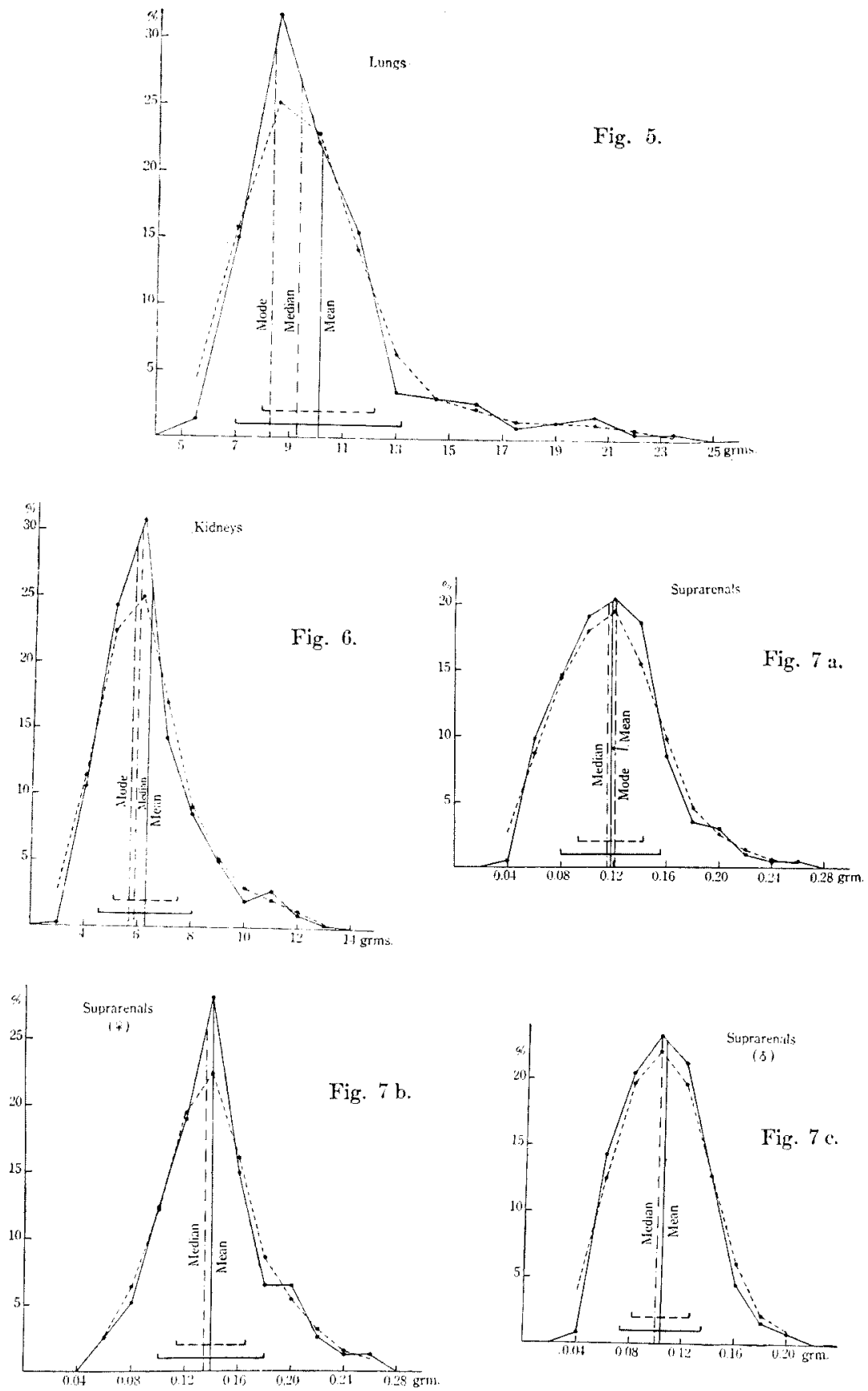
the extreme $1.39: 1$. In 46 cases, in which the right was heavier, the ratios (right: left) were 1.08:1 and 1.33:1 respectively. The sexual relation was not found in the ratio of the weight of each side kidney. The left was heavier in $60 \%$ of the 151 male dogs, and lighter in $20 \%$, and in the 91 female dogs the left was heavier in $65 \%$ and lighter in $16 \%$.

The relative weight of the suprarenal glands differed somewhat according to the sex of the animals, that is, in male dogs the glands are relatively smaller than in the female. This fact accords with the finding in the Wistar Institute on the albino rat..$^{6)}$ The data yielded in the hands of Koji$\mathrm{ma}$ on the suprarenals of normal rabbits (100 male and 100 female $)^{7}$ the reverse seeningly rather holds true, but the difference was not so large as in the present measurements.

Though some difference in the relative weight was seen between the fellow glands we can not attribute any significance to it, since in the great majority of cases the suprarenal glands were specially experimented on for another purpose.

The coefficient of variation was smallest for the heart $(15.0)$ and largest for the spleen (38.8). The accompanying small table consists of the coefficient of variation for the organs of dogs, arranged according to the magnitude and those for the rabbits of Brown and his co-workers. Notwithstanding that their data on rabbits are based on material large and uniform, and the present ones on dogs on material obtained under somewhat diverse conditions, the coefficient of variation for the various organs shows rather a similar figure.

\section{TABLE II.}

The coefficient of variation for the relative weight of organs.

$\begin{array}{lcc} & \begin{array}{c}\text { Dogs } \\ \text { (Sato) }\end{array} & \begin{array}{c}\text { Rabbits (Brown } \\ \text { and others, 1926) }\end{array} \\ \text { Heart } & 15.0 & 11.98 \\ \text { Liver } & 27.2 & 25.10 \\ \text { Kidneys } & 27.7 & 17.53 \\ \text { Lungs } & 30.6 & \\ \text { Pancreas } & 30.8 & \\ \text { Suprarenals } & 31.8 & 38.15 \\ \text { Spleen } & \text { (Fenale } 27.7, \text { male 29.8) } & \\ & 38.8 & 50.35\end{array}$

That the relative weight of the heart of dogs is similar in a paper of Junkersdorf and in the present may be due to the smallness of the coef-

6) Hatai, Am. J. of Anat., 1913(-14), 15, 108-9; Don altson, Am. J. of Physiol., $1928,67,1$.

7) Kojima, Tohoku J. Exp. Med., 1929, 13, Table II on p. 214. 
ficient of variation. The limits of the relative weight of the spleen, given in Ellenberger and $\mathrm{Baum}$ and in Junkersdorf are too narrow. Our daily experience on laparotomizing dogs rather points to a considerable variation in the size of the spleen. The narrow limits in Junkersdorf might be due simply to the number of animals being too small. The numerals in $\mathbf{K u r a k a m i}$ on the relative weight of the pancreas do not differ so greatly from ours.

\section{SUMMMARI :}

The relative weights of the organs from $272 \mathrm{dogs}$, which were well nourished, but employed in the epinephrine secretion experiment or the blood pressure tracing, and finally killed by bleeding, are treated in this paper.

The maximum, minimum, mean, median, mode, standard deviation, probable error and the coefficient of variation of the relative weight of the heart, spleen, liver, pancreas, lungs, kidneys and suprarenals are given in a table and figures. 